International Journal of Pure and Applied Mathematics

Volume 82 No. 5 2013, 825-828

ISSN: 1311-8080 (printed version); ISSN: 1314-3395 (on-line version)

url: http://www.ijpam.eu

doi: http://dx.doi.org/10.12732/ijpam.v82i5.11

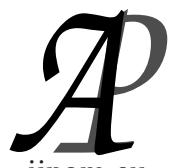

ijpam.eu

\title{
COMPOSED PENCILS ON A SMOOTH CURVE WITH A SINGULAR MODEL IN A QUADRIC SURFACE
}

\author{
E. Ballico \\ Department of Mathematics \\ University of Trento \\ 38123 Povo (Trento) - Via Sommarive, 14, ITALY
}

\begin{abstract}
Let $C$ be the normalization of an integral curve of type $\left(a, a^{\prime}\right)$ on $\mathbb{P}^{1} \times \mathbb{P}^{1}$. We give conditions on $\operatorname{Sing}(Y)$ and $y$ for the non-existence of a pencil on $C$ partially composed with the $g_{a}^{1}$ or the $g_{a^{\prime}}^{1}$ obtained in $C$ from the projections $\mathbb{P}^{1} \times \mathbb{P}^{1} \rightarrow \mathbb{P}^{1}$.
\end{abstract}

AMS Subject Classification: 14H51, 14H50

\section{Introduction}

Let $Q \subset \mathbb{P}^{3}$ be a smooth quadric surface. Let $\pi: Q \rightarrow \mathbb{P}^{1}$ and $\pi^{\prime}: Q \rightarrow \mathbb{P}^{1}$ be the two projections. We have $\operatorname{Pic}(Q) \cong \mathbb{N}^{2}$. We have $h^{0}\left(\mathcal{O}_{Q}(a, b)\right)=(a+1)(b+1)$ and $h^{1}\left(Q, \mathcal{O}_{Q}(a, b)\right)=0$ if $a \geq-1$ and $b \geq-1$. A curve $\left|\mathcal{O}_{Q}(a, b)\right|$ is said to have type $(a, b)$. The lines of $Q$ are the curve with either type $(1,0)$ or type $(0,1)$. We use the convention that the fibers of $\pi$ have type $(0,1)$, while the fibers of $\pi^{\prime}$ have type $(1,0)$. Fix an integral $Y \in\left|\mathcal{O}_{Q}\left(a, a^{\prime}\right)\right|$. Let $w: C \rightarrow Y$ be the normalization map. Let $m: C \rightarrow \mathbb{P}^{1}$ (resp. $m^{\prime}: C \rightarrow \mathbb{P}^{1}$ ) be the composition of $w$ with $\pi \mid Y$ (resp. $\left.\pi^{\prime} \mid Y\right)$. We have $\operatorname{deg}(m)=a$ and $\operatorname{deg}\left(m^{\prime}\right)=a^{\prime}$. Set $R:=m^{*}\left(\mathcal{O}_{\mathbb{P}^{1}}(1)\right)$

Received: December 18, 2012

(c) 2013 Academic Publications, Ltd. url: www.acadpubl.eu 
and $R^{\prime}:=m^{*}\left(\mathcal{O}_{\mathbb{P} 1}(1)\right) . \quad R$ and $R^{\prime}$ are spanned line bundles of degree $a$ and $a^{\prime}$, respectively. Under mild assumptions on $Y$ we have $h^{0}(R)=h^{0}\left(R^{\prime}\right)=2$ (see Remark 1). In this note we consider the following classical question. Under which assumptions on $Y$ there is no spanned $M \in \operatorname{Pic}(C), M \neq R$ and $M \neq R^{\prime}$, such that $h^{0}(C, M)=2$ and the morphism $\phi: C \rightarrow \mathbb{P}^{1}$ induced by $|M|$ is composed either with $m$ or with $m^{\prime}$, i.e. one of the two maps $(m, \phi)$ or $\left(m^{\prime}, \phi\right)$ from $C$ into $\mathbb{P}^{1} \times \mathbb{P}^{1}$ is not birational onto its image? Assume that $M$ exists and let $D$ be the normalization of either $(m, \phi)$ or $\left(m^{\prime}, \phi\right)$. Set $y:=\operatorname{deg}(M)$. The map $(m, \phi)$ (or $\left(m^{\prime}, \phi\right)$ induces a non-constant map $\beta: C \rightarrow D$ and $(m, \phi)$ (resp. $\left.\left(m^{\prime}, \phi\right)\right)$ factor though $w$. Set $b:=\operatorname{deg}(\beta)$. We have $b \mid y, b<y$; we have $b \mid a$ and $b<a$ (resp. $b \mid a^{\prime}$ and $b<a^{\prime}$ ). Since $M \notin\left\{R, R^{\prime}\right\}, C$ has genus $g \geq 2$ and $D$ has positive genus. In this note we prove the following result.

Theorem 1. Assume the existence of a spanned $M \in \operatorname{Pic}^{y}(C), M \neq R$ (resp. $M \neq R^{\prime}$ ) such that $\phi$ is composed with $m$ (resp. $\left.m^{\prime}\right)$, i.e. the map $(m, \phi)$ (resp. $\left.\left(m^{\prime}, \phi\right)\right)$ has degree $b \geq 2$ onto its image. We have $b \mid y$ and $b<y$. We have $b \mid a$ and $b<a$ (resp. $b \mid a^{\prime}$ and $b<a^{\prime}$ ). Let $\mathcal{J}$ the conductor of $C$, seen as an ideal sheaf of $Q$, with $\mathcal{O}_{Z}:=\mathcal{O}_{Q} / \mathcal{J}$. Then none of these conditions is satisfied:

1. $h^{1}\left(Q, \mathcal{J}\left(a-2, a^{\prime}-2-y / b\right)\right)=0\left(\operatorname{resp} . h^{1}\left(Q, \mathcal{J}\left(a-2-y / b, a^{\prime}-2\right)\right)=0\right)$;

2. $Y$ has only ordinary nodes and ordinary cusps as singularities, $\operatorname{Sing}(Y)$ is formed by general points of $Q$ and $\sharp(\operatorname{Sing}(Y)) \leq(a-1)\left(a^{\prime}-1-y / b\right)$ (resp. $\left.\sharp(\operatorname{Sing}(Y)) \leq(a-1-y / b)\left(a^{\prime}-1\right)\right)$;

3. assume $a^{\prime} \geq 2+y / b$ (resp. $a \geq 2+y / b$; set $v:=\max \left\{a-2, a^{\prime}-2-y / b\right\}$, $u:=\min \left\{a-2, a^{\prime}-2-y / b\right\} \quad$ (resp. $v:=\max \left\{a-2-y / b, a^{\prime}-2\right\}$ and $\left.u:=\min \left\{a-2-y / b, a^{\prime}-2\right\}\right)$. Set $\alpha:=\lfloor u / 3\rfloor$. Y has only ordinary nodes and ordinary cusps as singularities, no two of the points of $\operatorname{Sing}(Y)$ are contained in a line of $Q$, at most $u+v$ of the points of $Z$ are contained in a curve of type $(1,1)$ and at most $3 u+1$ of the points of $Z$ are contained in a curve of type $(2,1)$ or $(1,2)$ and $\sharp(\operatorname{Sing}(Y)) \leq v-u+10 \alpha-1$;

4. $Y$ has only ordinary nodes and ordinary cusps as singularities, $a^{\prime} \geq 2+y / b$ (resp. $a \geq 2+y / b)$ and $\sharp(\operatorname{Sing}(Y)) \leq \min \left\{a-1, a^{\prime}-1-y / b\right\} \quad(($ resp. $\left.\sharp(\operatorname{Sing}(Y)) \leq \min \left\{a-1-y / b, a^{\prime}-1\right\}\right)$.

We work over an algebraically closed field $\mathbb{K}$. 


\section{The Proof}

Let $Z \subset Q$ be a zero-dimensional scheme. Let $\Delta_{Z}$ be the union of all lines $L \subset Q$ such that $L \cap Z=\emptyset$. Notice that $\Delta_{Z}$ is a finite union of lines. This is a fundamental difference between $Q$ and $\mathbb{P}^{2}$.

Lemma 1. Fix $(x, v) \in \mathbb{N}^{2}$ and the ideal sheaf $\mathcal{J}$ of a zero-dimensional scheme $Z$ such that $h^{1}(\mathcal{J}(u, v))=0$. Fix a set $B \subset Q$ such that $B \cap \Delta_{Z}=\emptyset$ and there is an integer $b>0$ such that for each $I \in\left|\mathcal{O}_{Q}(0,1)\right|$ either $I \cap B=\emptyset$ or $\sharp(I \cap B)=b$. Set $y:=\sharp(B)$. Assume $b \leq u+1$. Then $Z \cap B=\emptyset, b \mid y$ and $h^{1}\left(\mathcal{I}_{\mathcal{J} \cup B}(u, v+y / b)\right)=0$.

Proof. Since $Z \subseteq \Delta_{Z}$ and $B \cap \Delta_{Z}=\emptyset$, we have $B \cap Z=\emptyset$. By assumption there is a curve $F \in\left|\mathcal{O}_{Q}(0, y / b)\right|, F$ union of $y / b$ distinct lines such that $B \subset F$ and $\sharp(F \cap I)=b$ for each connected component $I$ of $F$. Since $B \cap \Delta_{Z}=\emptyset$, we have $Z \cap F=\emptyset$. Hence $\operatorname{deg}((Z \cup B) \cap I)=b$ for each component $I$ of $F$. Since $b \leq u+1$, we get $h^{1}\left(F, \mathcal{I}_{Z \cup B}(u, v+y / b)\right)=0$. Since $Z=(Z \cup B) \backslash(Z \cup B) \cap F$. Use the exact sequence

$$
0 \rightarrow \mathcal{I}_{Z}(u, v) \rightarrow \mathcal{I}_{Z \cup B}(u, v+y / b) \rightarrow \mathcal{I}_{(Z \cup B) \cap F, F}(u, v+y / b) \rightarrow 0
$$

and the assumption $h^{1}(\mathcal{J}(u, v))=0$.

Remark 1. The adjunction formula gives that $h^{0}(R)=2\left(\right.$ resp. $h^{0}\left(R^{\prime}\right)=$ $2)$ if and only if $h^{1}\left(\mathcal{J}\left(a-2, a^{\prime}-3\right)\right)=0\left(\operatorname{resp} . h^{1}\left(\mathcal{J}\left(a-3, a^{\prime}-2\right)\right)=0\right)$. Just the existence of a reduced curve $Y \in\left|\mathcal{O}_{Q}\left(a, a^{\prime}\right)\right| \operatorname{implies} h^{1}\left(\mathcal{J}\left(a-2, a^{\prime}-2\right)\right)=0$.

Proof of Theorem 1. We first assume that $|M|$ is composed with the $g_{a}^{1}$ on $C$ induced by $\pi$. Fix a general $A \in|M|$ and set $B:=w(A) \subset Y$. Since $|M|$ is a complete linear system, even in positive characteristic we see that $\phi$ is not composed with a Frobenius. Hence $\phi$ is separable. Since $\phi$ is separable and $A$ is general in $|M|, A$ is formed by $y$ distinct points. Since $|M|$ is spanned and $A$ is general, we have $A \cap w^{-1}(\operatorname{Sing}(Y))=\emptyset$. Hence $B \subset Y, B \cap Z=\emptyset$ and $\sharp(B)=y$. Since $|M|$ has no base point, $\Delta_{Z}$ is a finite union of lines and $A$ is general, we have $B \cap \Delta_{Z}=\emptyset$. Fix $O \in A$. Since $M$ has no base points, we have $h^{0}\left(C, \mathcal{O}_{C}(A \backslash\{O\})\right)=h^{0}\left(C, \mathcal{O}_{C}(A)\right)-1$, i.e. $h^{0}\left(C, \omega_{C}(-A)\right)=$ $h^{0}\left(C, \omega_{C}(-A)(O)\right)$ (Riemann-Roch and Serre duality). The adjunction formula gives $\omega_{Y} \cong \mathcal{O}_{Y}\left(a-2, a^{\prime}-2\right)$. Since $h^{i}\left(\mathcal{O}_{Q}(-2,-2)\right)=0, i=0,1$, we get that $\left|\omega_{C}\right|$ is induced by the linear system $\left|\mathcal{J}\left(a-2, a^{\prime}-2\right)\right|$ on $Q$. Since $A \cap$ $w^{-1}(\operatorname{Sing}(Y))=\emptyset$, we get $h^{1}\left(C, \omega_{C}(-A)\right)=h^{1}\left(\mathcal{I}_{Z \cup B}\left(a-2, a^{\prime}-2\right)\right)$. Hence $h^{1}\left(\mathcal{I}_{Z \cup B}\left(a-2, a^{\prime}-2\right)\right)>0$ Since $h^{1}\left(\mathcal{I}_{Z \cup B}\left(a-2, a^{\prime}-2\right)\right)>0$, lemma 1 gives a contradiction. 
Now assume that $|R|$ is composed with the $g_{a^{\prime}}^{1}$ induced by $\pi^{\prime}$. We conclude as above taking $a^{\prime}$ instead of $a$.

Hence we proved Theorem 1 under the assumption (1). We only need to prove that in the remaining cases the assumption of (1) is satisfied. If $Y$ has only ordinary nodes and ordinary cusps, then $Z$ is the set $\operatorname{Sing}(Y)$ with its reduced structure. In case (2) uses the definition of "general set". In case (3) use [1], lemma 2. The proof that (4) implies (1) is an easy exercise.

\section{Acknowledgements}

The author was partially supported by MIUR and GNSAGA of INdAM (Italy).

\section{References}

[1] E. Ballico, On the gonality sequence of smooth curves: normalizations of singular curves in a quadric surface, Preprint. 